\title{
THE CHARACTERIZATION OF MYCOBACTERIAL STRAINS BY THE COMPOSITION OF THEIR LIPIDE EXTRACTS*
}

\author{
By D. W. Smith $\dagger$, H. M. Randall $\ddagger$, M. M. Gastambide-Odier $\dagger$, \\ and A. L. Koevoet $\ddagger$ \\ Department of Medical Microbiology, Universily of Wisconsin, Madison, Wis. \\ and \\ Harrison M. Randall Laboratory of Physics, Unizersily of Michigan, Ann Arbor, Mich.
}

After a brief introduction, we shall present studies on the reproducibility of the techniques, a consideration of the sensitivity of the method and, finally, an application that is presently under consideration.

In 1948 a research program studying the immunizing properties of fractions of the tubercle bacillus was in progress in the Bacteriology Department of the University of Michigan. In this study, complex extracts were prepared from mass cultures of the organism and were tested in guinea pigs and mice for their capacity to cause alterations in the normal resistance of the animals to tuberculosis.

About this time, one of us (HMR) approached the Bacteriology Department with an inquiry as to whether there were any lines of research in which infrared spectroscopic studies could make a contribution to the study of complex biological mixtures. The feasibility of such an investigation in the work on immunity in tuberculosis was evident. The fact that the extracts being prepared from the tubercle bacillus were of a lipide nature proved to be an important factor in the progress made in the present research.

\section{Studies on Reproducibility}

After several abortive attempts to find a correlation between the immunizing potency of a given preparation and its spectrum, we decided that it would first be necessary to study the system with which we were working to determine the degree of reproducibility of bacterial extracts prepared under similar conditions and the extent to which the different variable factors could be altered without affecting the extracts.

Working with total $\mathrm{CHCl}_{3}$-soluble fractions and subfractions prepared by precipitation with acetone, we were able to show ${ }^{1}$ that, with sufficient care, different lots of tubercle bacilli grown and extracted under similar conditions yielded substances, the infrared spectra of which were sufficiently reproducible to warrant further study. Following this, a study was made of the effects of age of the inoculum, age of culture, temperature of incubation, composition of the medium, and duration of extraction on the reproducibility of the infrared spectra of the lipide extracts. It was determined that all of these factors were critical and that, if varied, they would lead to changes in the composition of the lipide mixtures.

* This work was supported by grants from the Research Corporation, New York, N. Y., the Wisconsin Anti-Tuberculosis Association, Milwaukee, Wis., and the Rackham Fund of the Graduate School, University of Michigan, Ann Arbor, Mich.

$\dagger$ University of Wisconsin.

$¥$ University of Michigan. 


\section{Sensitivity}

Having chosen a set of conditions that would lead to spectroscopically reproducible lipide extracts, it was decided, as a measure of the sensitivity of the method, to determine whether it could distinguish between strains known to be biologically distinct. The following groups of organisms were chosen so that each succeeding study involved a more subtle biological difference and, possibly, a more subtle chemical difference.

(1) The first study was concerned with two strains, H37Rv and H37Ra, derived from a single parent culture, one being virulent and the other avirulent. The cultures can be distinguished grossly.

(2) As a second phase of the study, human, bovine, and avian varieties of the tubercle bacillus were examined. The human and bovine varieties are very similar, differing only in the fact that bovine bacilli produce progressive disease in rabbits while human bacilli do not. Both produce progressive tuberculosis in guinea pigs and in man.

(3) Next to be examined were recently isolated strains of human tubercle bacilli. We were looking for chemically distinct subgroups, since there are no recognized biological subgroups of the human variety of bacillus.

(4) The last phase of the work that will be discussed, the one now under investigation, is concerned with a study of differences in lipide composition between strains of mycobacteria isolated from human sputum; some of these, namely tubercle bacilli, are capable of producing disease in guinea pigs, while others do not have this property. The latter strains are called atypical acidfast bacilli.

Throughout the course of this study improvements have been made in the manner of recording spectra, in the methods of preparing extracts, and in their subsequent resolution into simpler mixtures and single compounds. For this reason, details of the methods of separation used in the early studies will not be presented here.

For the study of $\mathrm{H} 37 \mathrm{Rv}$ and $\mathrm{H} 37 \mathrm{Ra},{ }^{2}$ identical procedures were carried out on the two strains. Mass cultures of both strains were prepared on a synthetic medium, the cells were harvested at six weeks, and extracted with a mixture of equal parts of methanol and $\mathrm{CHCl}_{3}$. This extract was filtered to remove the cells and then was cooled to $5^{\circ} \mathrm{C}$. The white solid that precipitated was removed and dried at the same temperature. The infrared spectra of the $5^{\circ} \mathrm{C}$. insoluble fractions from both strains are shown for comparison in FIGURE 1. The differences have been shown to be reproducible, and they indicate chemical differences in the two strains, either qualitative or quantitative, or both.

Turning next to a study of possible differences in lipide composition between human and bovine tubercle bacilli, ${ }^{3}$ mass cultures were prepared and extracted under conditions similar to those mentioned in the work on the $\mathrm{H} 37$ strains. This time the methanol-chloroform extract was precipitated at $5^{\circ}$ and the filtrate was again precipitated at $-30^{\circ} \mathrm{C}$. In FIGURE 2 are shown for comparison the infrared spectra of whole cells of human and bovine bacilli (upper two spectra) and the total lipide extracts (lower two spectra). Although minor spectral differences exist, they are not sufficiently reproducible to be of help in distinguishing the strains. In FIGURE 3 are shown the spectra of the $5^{\circ}$ and 

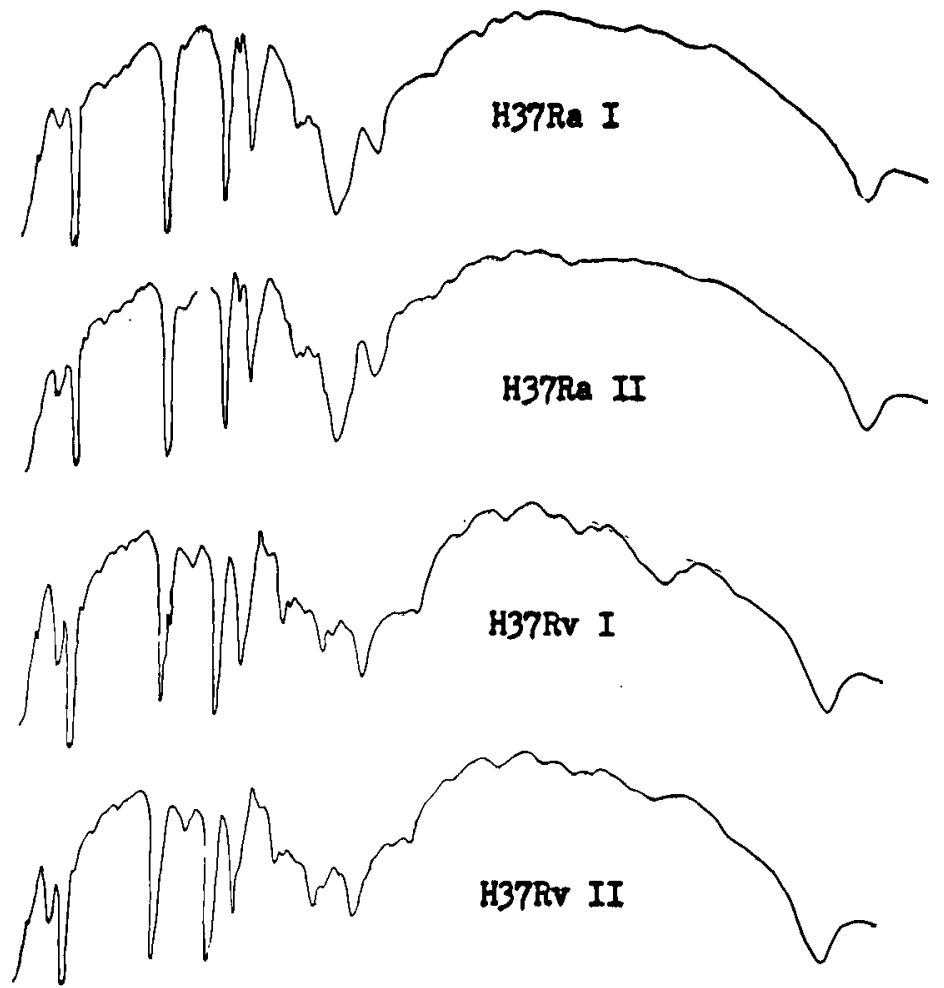

Figure 1. Infrared spectra of the $5^{\circ} \mathrm{C}$. insoluble fractions of $\mathrm{H} 37 \mathrm{Rv}$ and $\mathrm{H} 37 \mathrm{Ra}$. Lot I of each strain was harvested in March of 1951, lot II in May of 1951.

$-30^{\circ} \mathrm{C}$. insoluble fractions for a human and bovine strain. The $5^{\circ}$ fractions have proved to be very reproducible, but no significant spectral differences can be seen. The $-30^{\circ} \mathrm{C}$. fraction lacks the same degree of reproducibility, and it was only after a large number of these fractions had been prepared and spectra recorded that the minor band at $1510 \mathrm{~cm}^{-1}(6.63 \mu)$ was observed to be uniformly present in the fractions from bovine strains and not in the corresponding fraction from human strains.

Substantial masses of the $-30^{\circ} \mathrm{C}$. insoluble fraction of two bovine and one human strain were prepared and subjected to further separation by batch adsorption chromatography on Magnesol*. The fractions eluted from the adsorbent in ether are shown in FIGURE 4 . It can be seen that a considerable concentration of the compound responsible for the $1510 \mathrm{~cm} .{ }^{-1}$ band has occurred in the case of the lipides of the two bovine strains, while none of this material can be detected in the fractions of the human-strain lipides. Subsequently this substance was demonstrated to be a single compound present in the lipides of seven bovine strains and absent in the lipides of twenty human strains.

The next phase of this study was concerned with an attempt to detect

* Magnesol-magnesium silicate, Westvaco Chemical Co., Chlor-Alkali Div., South Charleston, West Va. 

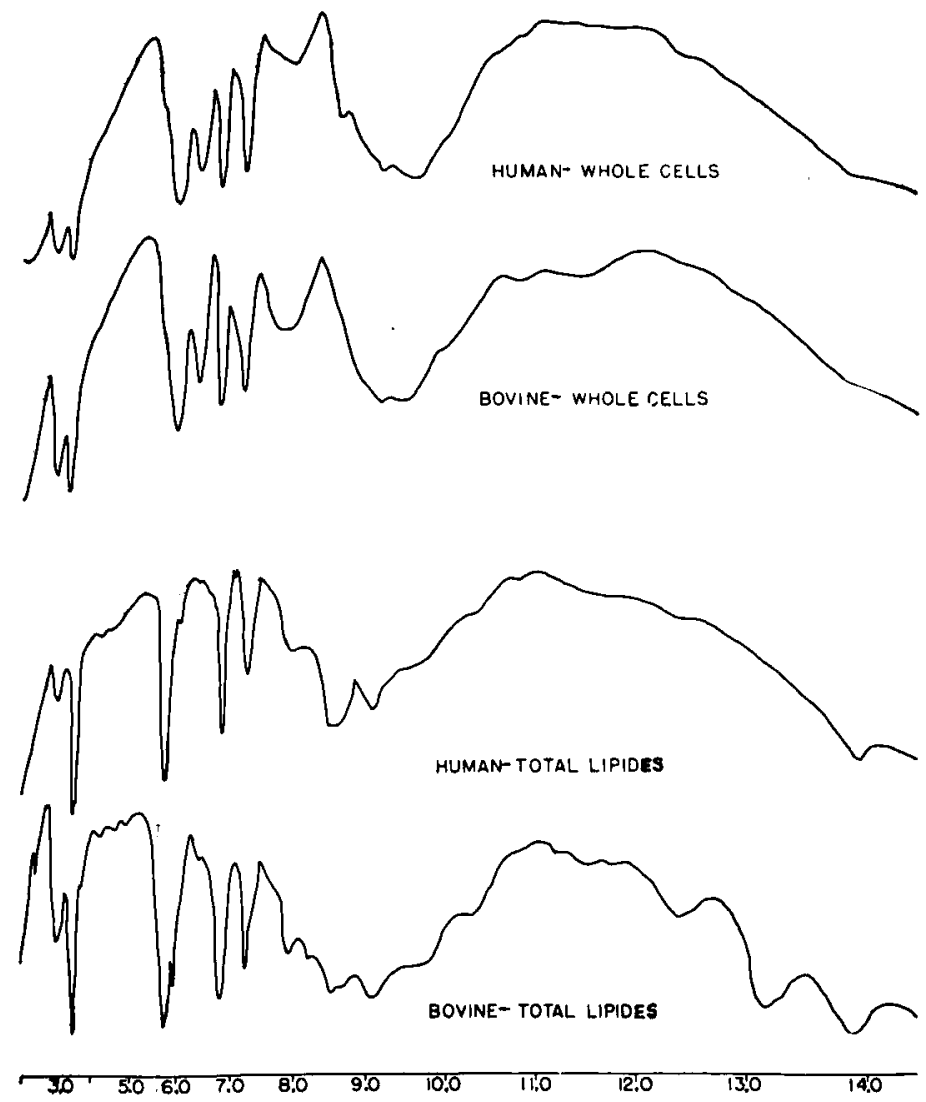

Figure 2. Infrared spectra of the whole cells (Nujol mull) and total lipides of human and bovine tubercle bacilli.

chemical differences between strains of human tubercle bacilli recently isolated from the sputum of patients. ${ }^{4}$ At this time we began employing column chromatography to achieve better separations. Cultures were extracted with a mixture of equal parts of ethanol and diethyl ether, and the extract was partitioned between ether and water to remove the glycerol. Twenty cultures have been prepared and extracted in this manner and the extracts chromatographed on Magnesol-Celite* . For the most part the results indicate a similarity of lipide composition. In only one strain was a compound found to be absent in the ether-soluble lipides when it was known to represent 15 per cent of the lipides of all other human strains. Even in this one strain, the missing compound could be demonstrated in the lipides later removed from the strain in $\mathrm{CHCl}_{3}$.

Examples of the types of substances found in the human tubercle bacillus are shown in FIGURES 5 and 6 . Because of the rather uniform distribution in the

* Celite-Analytical Filter Aid, Johns Manville Co., New York, N. Y. 

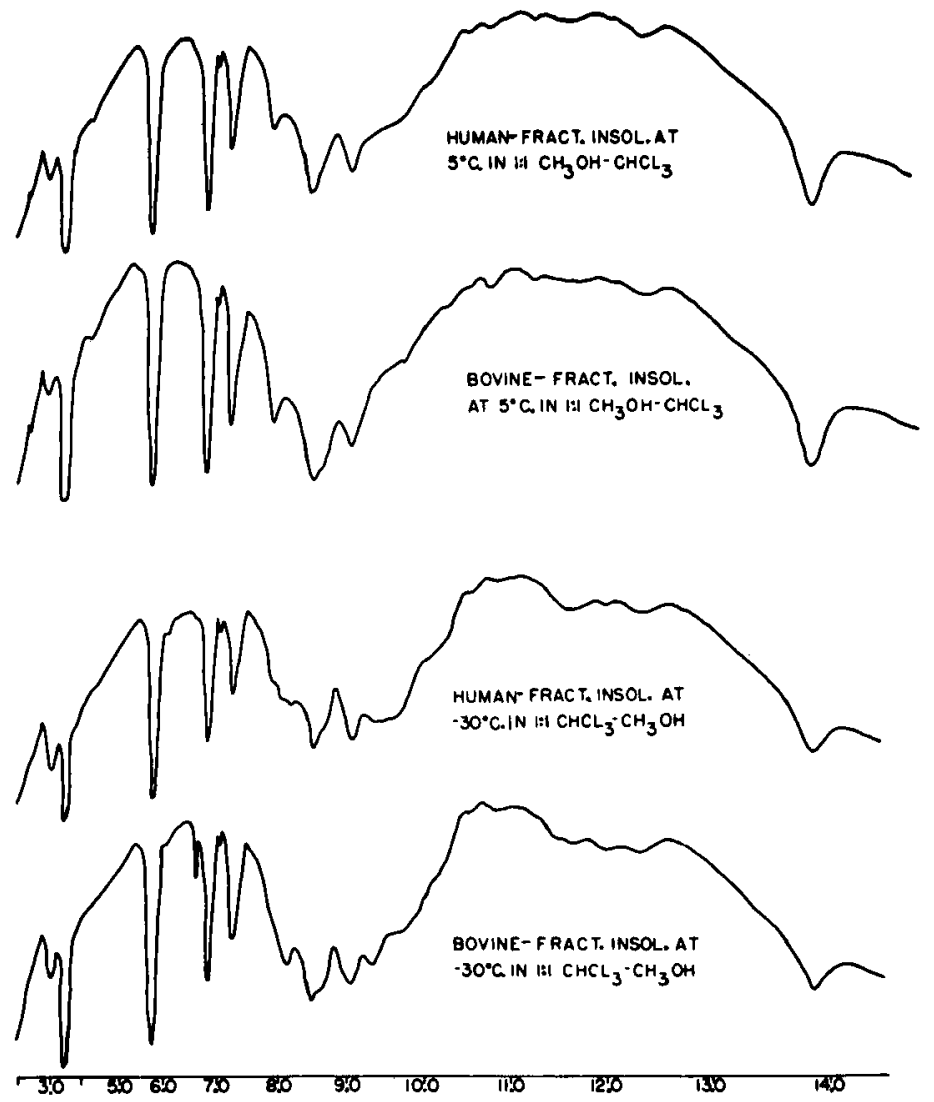

Figure 3. Spectra of the $5^{\circ} \mathrm{C}$. and $-30^{\circ} \mathrm{C}$. insoluble fractions prepared from the lipides of human and bovine tubercle bacilli.

various types of mycobacteria of the series of compounds shown in FIGURE 5 , only limited chemical studies have been made. On the basis of chromatography, of saponification products, and of comparison of the spectra with spectra of known materials, compound A would appear to be a triglyceride, compound $\mathrm{C}$ a diglyceride, and compound $\mathrm{D}$ a monoglyceride. The spectrum of the fatty acid shown is only a representative of the group of fatty acids present in lipides of mycobacteria. This group is resolved, by chromatography on rubber columns, in to the following: palmitic, stearic, oleic, and tuberculostearic acids, and others only partially separated.

The compounds shown in FIGURE 6 are not as uniformly distributed in all of the human strains. Mycolic acid, empirical formula $\mathrm{C}_{87} \mathrm{H}_{174} \mathrm{O}_{4}$, and dimycocerenate of phthiocerol, empirical formula $\mathrm{C}_{95} \mathrm{H}_{188} \mathrm{O}_{5}$, were identified by comparison of their spectra with spectra of the pure compounds isolated from mycobacteria by Asselineau and Lederer. ${ }^{5}$ Cord factor, a toxic glycolipide, empirical formula $\mathrm{C}_{186} \mathrm{H}_{366} \mathrm{O}_{17}$, was identified by comparison of spectra and melting points with the pure compound isolated by Noll. ${ }^{6}$ 

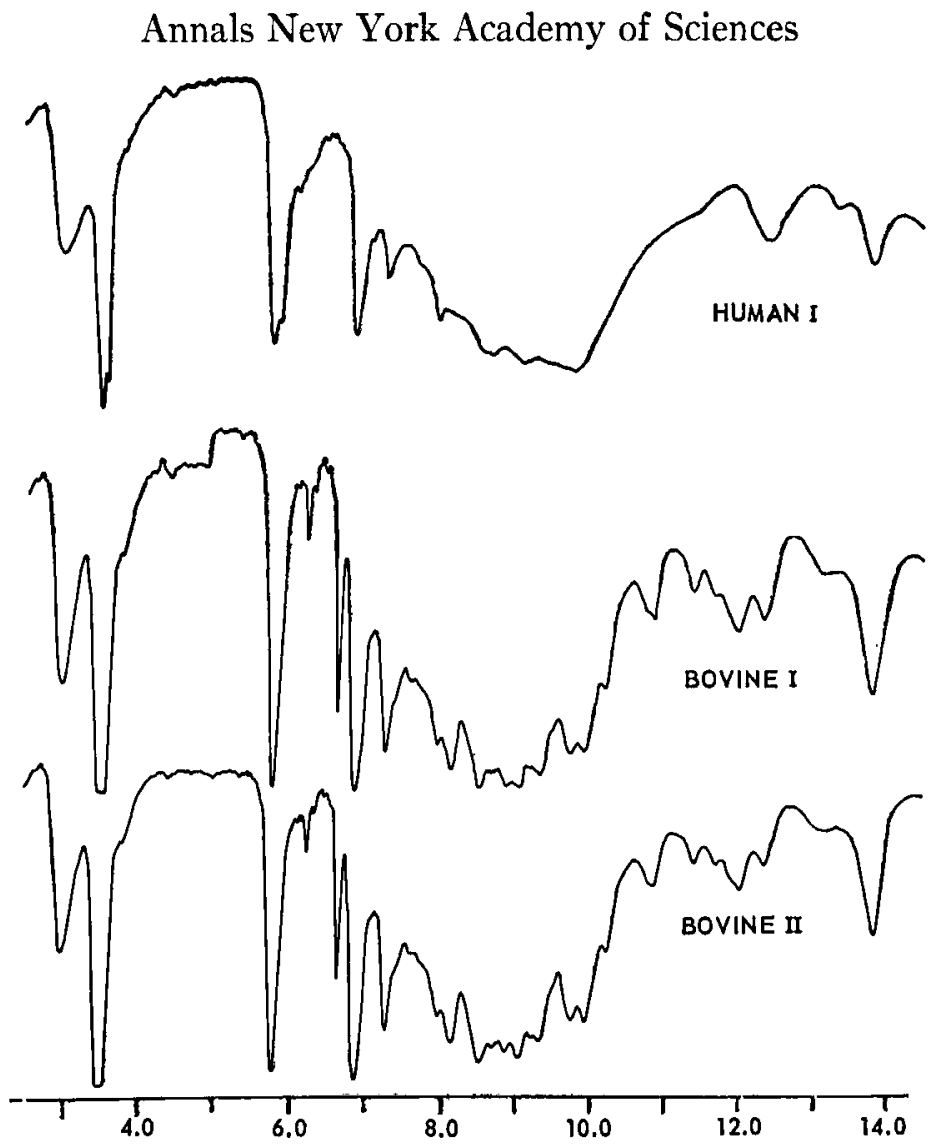

Figure 4. Spectra of the ether eluates separated by batch adsorption from the $-30^{\circ} \mathrm{C}$. insoluble fractions of two bovine cultures and one human culture.

We should like to give primary emphasis to the work now in progress, in which we are studying differences in lipide composition between human tubercle bacilli and the so-called atypical acid-fast bacilli. These organisms are similar to tubercle bacilli in many respects: they are acid-fast, and are repeatedly isolated from sputum or from surgical-resection material from patients ill with a disease similar in all respects to tuberculosis. However, they do not produce progressive disease in guinea pigs - a criterion that previously had been used to decide whether or not a given strain was tubercle bacillus or not. There are as yet no recognized biological tests for the atypical strains.

A series of seven atypical cultures was obtained from recognized sources and, at the time of receipt, all the cultures were said to fall into the atypical classification. These strains, together with other known varieties of acid-fast bacilli (BCG, Mycobacterium phlei, avian, bovine, and human strains), were cultured on a synthetic medium for six weeks at $37^{\circ} \mathrm{C}$. The cultures were harvested, and the medium was withdrawn, autoclaved, and discarded. The cell masses of 25 one-gallon jug cultures of each strain were combined in a mixture of equal 


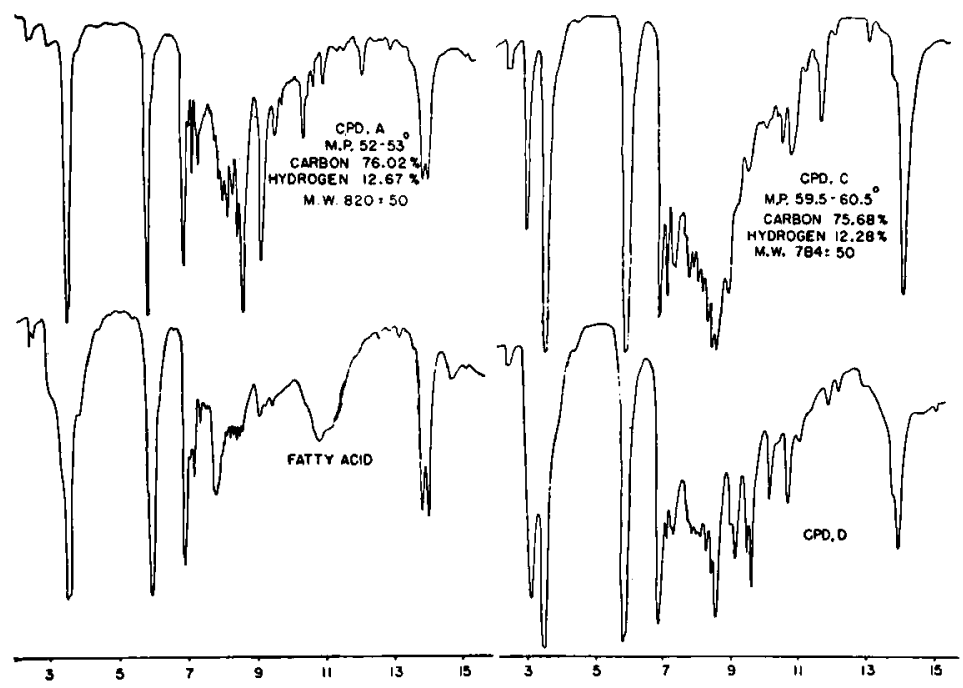

Figure 5. Infrared spectra of four substances found to be present in various types of mycobacteria.

parts ethanol and ether and were extracted for 48 hours. A second 24-hr. extraction with fresh ethanol-ether followed. The cells were separated from the solvent by filtration, were allowed to dry, and then were extracted for 24 hours with $\mathrm{CHCl}_{3}$. The ethanol-ether extract was concentrated to a minimum volume and then partitioned between water and ether. The ether-soluble fraction was dried over magnesium sulfate, the ether was removed under vacuum, and the dry lipide was stored at $5^{\circ} \mathrm{C}$. until it was chromatographed. The flow diagram for these operations is shown in CHART 1 .

Chromatography was carried out in $50 \mathrm{~mm}$.-diameter columns packed with $85 \mathrm{gm}$. of a mixture of equal parts Magnesol and Celite that had been washed with acetic acid, hot distilled water, ether, and methanol, and then activated at $150^{\circ} \mathrm{C}$. for $30 \mathrm{~min}$. The lipide material was applied to the column in hexane, the solvent used to make the slurry with which the column was packed. The columns were eluted sequentially with a series of solvents of increasing polarity, beginning with washed redistilled hexane and ending with mixtures of acetic acid in ether. Columns were treated as flowing chromatograms, and the eluates, $250 \mathrm{cc}$. each, were collected as they flowed from the column. The quantity of each solvent used varied according to the amount of material found in the eluates. The eluates were evaporated to dryness and weighed, and the infrared spectra were recorded on a Model 21 Perkin-Elmer doublebeam spectrophotometer.

TABLE. 1 gives the main compounds found in the different strains studied. The fatty acids and esters of fatty acids common to all strains are not includerl. Spectra of some of these substances are to be found in FIGUREs 5 and 6 . Free mycolic acid or its analogues and cord factor are found in most, but not all, of the different types of bacilli. 

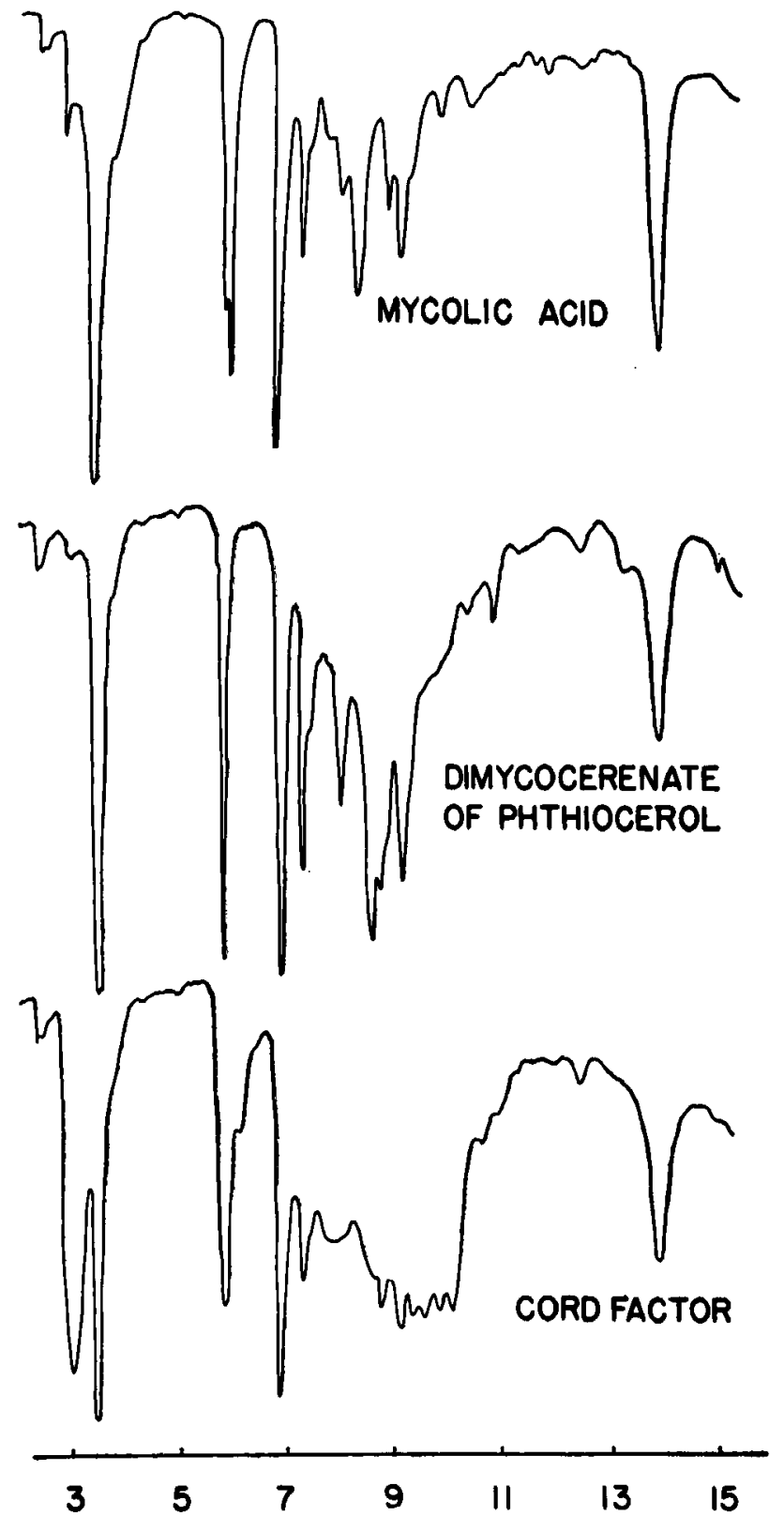

FIGURE 6. Infrared spectra of three compounds identified in the lipides of some cultures of the human variety of tubercle bacillus. 


\section{Chart 1}

Flow Diagram for the Extraction of Lipides of the Tubercle Bacillus

Cultures grown 6 weeks at $37^{\circ} \mathrm{C}$. (modified Long medium)

Media removed under vacuum; cells allowed to stand in $1: 1$ ethanolether for 48 hours, then collected on Buchner funnel

Original ether-ethanol extract evaporate to dryness

Cell mass

Re-extract with ethanol-ether for 24 hours

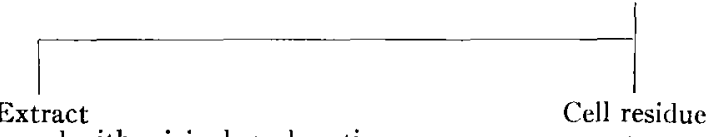

Concentrate to dryness, pool with original, and partition between water and ether; chromatograph the ether-soluble fraction

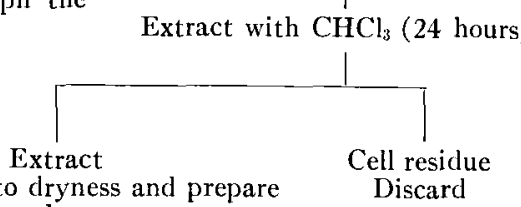

Concentrate to dryness and prepare

Discard for chromatography

TABLE 1

Lipide Substances Isolated from Various Strains of Mycobacteria

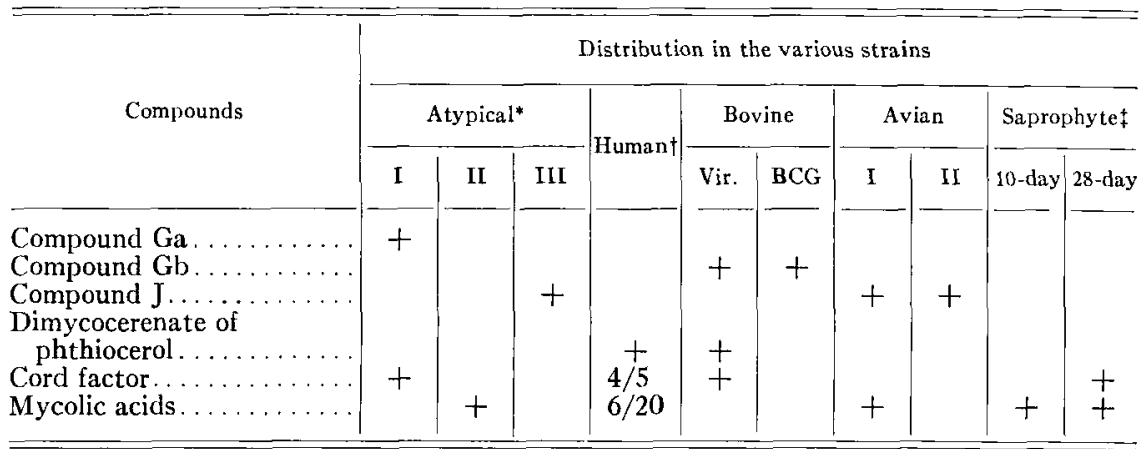

* Four strains in group I, two in group II, and one in group III.

$\dagger$ Twenty virulent human strains.

$\ddagger M$. phle $i$ harvested one time after 10 days' incubation and a second time after 28 days' incubation. 
Since cord factor and mycolic acid are so widely distributed in the various strains, they cannot be used generally as a means of differentiation. There are among the isolated substances three compounds of particular significance in classifying these strains: dimycocerenate of phthiocerol (DIM) and two unidentified compounds referred to as Ga and Gb. DIM, shown in FIGURE 6, has thus far been found only in human and bovine strains and represents approximately 15 per cent of the total lipides. Compound $\mathrm{Gb}$ has been found only in bovine strains and is the compound referred to earlier in discussing bovine tubercle bacilli. The isolation of a spectroscopically identical compound from a bovine strain was confirmed by Noll. ${ }^{7}$ Compound $\mathrm{Ga}$ has been found repeatedly in the lipides of some of the atypical strains. Two of these strains, obtained from different sources and differing somewhat in growth characteristics, have been cultured 3 times each, and the extracts have been chromato-

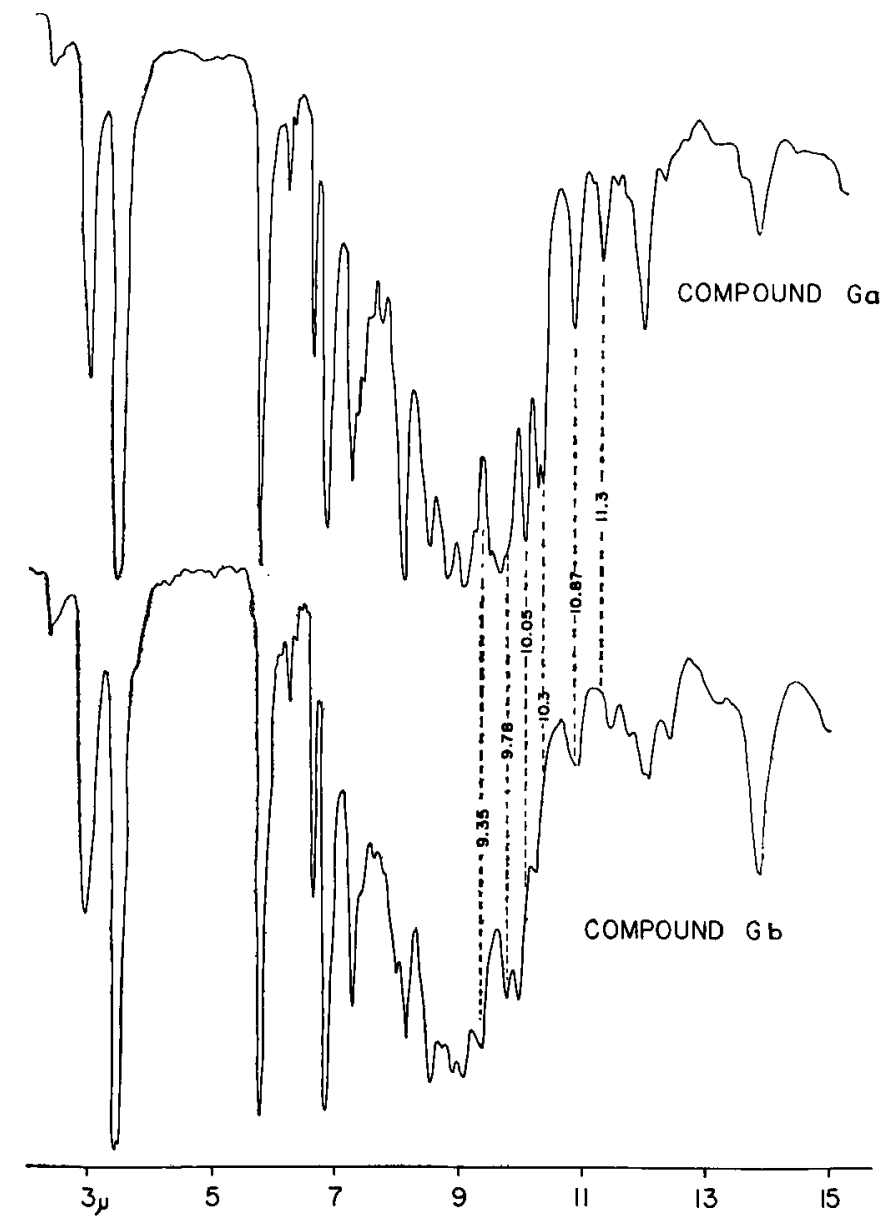

Figure 7. Comparison of the spectra of compounds $\mathrm{Ga}$ and $\mathrm{Gb}$. 
graphed from 5 to 8 times with the demonstration of compound $\mathrm{Ga}$ on each occasion. Compound Ga could not be detected in the lipides of 3 of the 7 strains originally labeled atypical. Concerning 2 of these cultures lacking this substance (see TABLE 1, atypical group II), after this study was completed, it was suggested by the investigator from whom the cultures were received that, since these cultures were isolated frequently in routine cultures and have never been identified in the tissues, they should probably be considered saprophytes. Our findings are in agreement with this suggestion. The third strain of the atypical group, lacking $\mathrm{Ga}$, has been found to contain a different substance, designated J, which otherwise has been found only in the 2 avian strains. Additional studies will be required to establish the significance of $\mathrm{J}$.

Thus far it would appear that we have isolated two substances, $\mathrm{Ga}$ and $\mathrm{Gb}$, the spectra of which are shown for comparison in FIGURE 7, which may be specific for the types of bacilli from which they were isolated. This possibility is made even more interesting by the fact that the infrared spectra of the two substances indicate a rather close chemical relationship. It is possible that the study of other atypical strains will lead to the recognition of other types and that each subgroup might be identified by a compound or group of compounds.

In TABLE 2 we have summarized the present information about $\mathrm{Ga}$ and $\mathrm{Gb}$. Both are fairly resistant to saponification, being split completely only after $72 \mathrm{hr}$. at $80^{\circ} \mathrm{C}$. In each case, the saponification products are an acid and an alcohol.

If the data that we have obtained thus far are confirmed, it would appear that it is possible to distinguish between each of the major subgroups of mycobacteria examined. The different varieties of mycobacteria could be grouped first on the basis of the presence or absence of DIM. Human and bovine strains, both containing DIM, could be separated by the presence of Gb in bovine strains only. The subgroups lacking DIM include atypicals, BCG, avian strains, and saprophytes. The atypical strains could be recognized by the presence of $\mathrm{Ga}, \mathrm{BCG}$ strains by the presence of $\mathrm{Gb}$, avian strains by the presence of $\mathrm{J}$, while the saprophyte strains contain none of the specific compounds. These observations are summarized in TABLE 1.

TABLE 2

Comparison of the Chemical and Physical Properties of Compotndes Ga, Gb and Their Alcoholic Derivatives

\begin{tabular}{|c|c|c|c|c|}
\hline & \multicolumn{4}{|c|}{ Compound } \\
\hline & $\mathrm{Ga}$ & $\mathrm{G}^{\prime} \mathrm{a}$ & Gb & $\mathrm{G}^{\prime} \mathrm{b}$ \\
\hline $\begin{array}{l}\text { Melting point .... } \\
\text { Carbon }(\%) \\
\text { Hydrogen }(\%) \ldots \\
\text { Nitrogen } \ldots \ldots \\
\text { Mol, weight . . . . } \\
\text { U.V. maxima. } \\
\text { Type of compound }\end{array}$ & $\begin{array}{c}\overrightarrow{72.5} \\
11.2 \\
\text { absent } \\
1000 \\
275,282 \\
\text { ester }\end{array}$ & $\begin{array}{c}- \\
64.2 \\
9.77 \\
\text { absent } \\
830 \\
275,282 \\
\text { alcohol }\end{array}$ & $\begin{array}{c}\overline{77.1} \\
12.1 \\
\text { absent } \\
1000 \\
275,282 \\
\text { ester }\end{array}$ & $\begin{array}{c}40^{\circ} \mathrm{C} \\
67.2 \\
10.8 \\
\text { absent } \\
800 \\
275,282 \\
\text { alcohol }\end{array}$ \\
\hline
\end{tabular}




\section{Discussion}

As far as the present work is concerned, we feel that the next step is to extend our findings to include a greater number of atypical strains. We should then like to develop a simple means of isolating $\mathrm{Ga}$, or its equivalents if subgroups of atypicals exist, so that its presence or absence in a given strain could be determined quickly. Ultimately, if this test is to be a practical means of determining whether a strain is atypical or not, it should be developed in a manner to eliminate the time-consuming expensive steps, including mass-culture preparation, chromatography, and spectroscopy. It might be possible to take advantage of specific chemical groups of Compound $\mathrm{Ga}$ in devising a color development test that could be performed on the patient's primary sputum culture.

It would be of interest to determine whether the preliminary results on classification of the various other groups of mycobacteria are still valid after more strains have been examined. Since the mycobacteria are not as well classified by immunological means as other groups of bacteria, a grouping based on lipide content might serve a useful purpose. Moreover, where a strain-specific substance, such as $\mathrm{Gb}$, is isolated, it would be interesting to determine whether the substance plays any role in the biological differences observed among the varieties of tubercle bacilli involved.

Thus far, this research has been entirely on a qualitative basis; that is, a compound is either present or not present. Quantitative studies might show significant differences between strains on the basis of relative amounts of some of the substances now found to be present in all strains.

We have made only very limited studies of bacteria outside the genus Mycobacterium. We compared the spectra of the whole cell and of a total lipide extract for each of eighteen types of bacteria* and found considerable specificity to the spectrum of the extract and less specificity to the spectrum of the whole-cell preparations. We see little hope for the use of infrared spectroscopy as a practical means of identifying bacteria in general, particularly when the method must compete with the highly specific techniques involving the use of fluorescent antibody.

Speaking in more general terms, we feel that we have demonstrated the utility of infrared spectroscopy as applied to the detection of differences in lipide composition among complex biological materials. Although the mycobacteria have been extremely valuable for study because of their high lipide content, it is possible that the techniques employed in this research could be useful in comparing, for example, normal and diseased tissues.

\section{Acknoweledgment}

We acknowledge our indebtedness to Paul Maker, Richard Putney, and Harold Patterson for their valuable assistance in this work.

* Including members of the following genera: Neisseria, Bacillus, Escherichia, Proteus, Staphylococcus, Aerobacter, Klebsiella, Micrococcus, and Pseudomonas. 


\section{Smith et al.: Characterization of Mycobacterial Strains}

\section{Addendum}

Since this paper was submitted for publication, additional atypical strains have been studied, bringing the total now to 13 strains. All of the new strains fit into the chemical classification developed in the earlier studies.

In addition, progress has been made on the chemical structure of the strain-specific compounds $\mathrm{Ga}, \mathrm{Gb}$, and $\mathrm{J}$. $\mathrm{Ga}$ and $\mathrm{Gb}$ appear to be closely related to the dimycocerenate of phthiocerol (DIM). The main points of difference are that the G compounds contain only one mycocerosic acid unit and the methoxyl group of DIM is absent in the G compounds, which contain instead a substituted aromatic ether. The $G$ compounds also contain a free $\mathrm{OH}$ group. Compound $\mathbf{J}$ has now been resolved into two closely related but spectroscopically distinct compounds, one of which is characteristic for avian tubercle bacilli and the other characteristic for the group III atypical strains.

\section{References}

1. Randall, H. M., D. W. Smith, A. Colm \& W. J. Nungester. 1951. Correlation of biologic properties of strains of $M y c o b a c t e r i u m$ with their infrared spectrums. I. $R e$ producibility of extracts of $M$. tuberculosis as determined by infrared spectroscopy. Am. Rev. Tuberc. 63: 372.

2. Randall, H. M., D. W. Smith \& W. J. Nungester. 1952. Correlation of biologic properties of strains of Mycobacterium with their infrared spectrums. II. The differentiation of two strains, $\mathrm{H} 37 \mathrm{Rv}$ and $\mathrm{H} 37 \mathrm{Ra}$ of $M$. tuberculosis by means of their infrared spectrums. Am. Rev. Tuberc. 65: 477.

3. Smith, D. W., W. K. Harrell \& H. M. Randall. 1954. Correlation of biologic properties of strains of Mycobacterium with their infrared spectrums. III. The differentiation of bovine and human varieties of $M$. tuberculosis by means of infrared spectrums. Am. Rev. Tuberc. 69: 505.

4. Kubica, G. P., H. M. Randale \& D. W. Smith. 1956. Correlation of biologic properties of strains of Mycobacterium with their infrared spectrums. IV. Fractionation and comparison of the lipids of human strains of Mycobacterium tuberculosis by means of their infrared spectrums. Am. Rev. Tuberc. Pulmonary Diseases. 73: 529.

5. Asselineau, J. \& E. Lederer. 1953. Chimie des lipides bactériens. Progr. chim. substances org. nat. $10: 170$.

6. Nols, H. 1956. The chemistry of cord factor, a toxic glycolipid of $M$. tuberculosis. Fortschr. Tuberkulose Forsch. 7: 149.

7. NoLL, H. 1957. The chemistry of some native constituents of the purified wax of $M y c o-$ bacterium tuberculosis. J. Biol. Chem. 224: 149. 